\title{
Circulating microRNA-122 as a potential biomarker for liver injury
}

\author{
XIANFENG DING $^{1 *}$, JIANV DING $^{1 *}$, JING NING $^{3,5,6}$, FAN YI $^{2}$, JIANKUI CHEN $^{5}$, DEYAO ZHAO $^{1}$, \\ JIAPING ZHENG ${ }^{4}$, ZICAI LIANG ${ }^{2}$, ZHIYUAN HU ${ }^{3}$ and QUAN DU ${ }^{2,7}$
}

\author{
${ }^{1}$ School of Life Science, Zhejiang Sci-Tech University, Hangzhou, Zhejiang 310018; ${ }^{2}$ Institute of Molecular Medicine, \\ Peking University, Beijing 100871; ${ }^{3}$ State Key Laboratory of Proteomics, Beijing Proteome Research Center, Beijing 102206; \\ ${ }^{4}$ Zhejiang Cancer Hospital, Hangzhou, Zhejiang 310022; ${ }^{5}$ Department of Laboratory Medicine, Affiliated Hospital, \\ Academy of Military Medical Sciences, Beijing 100071; ${ }^{6}$ Department of Laboratory Medicine, Beijing Municipal \\ Corps Hospital, Chinese People's Armed Police Forces, Beijing 100027; ${ }^{7}$ The State Key Laboratory of Natural \\ and Biomimetic Drugs, School of Pharmaceutical Sciences, Peking University, Beijing 100191, P.R. China
}

Received October 19, 2011; Accepted February 3, 2012

DOI: $10.3892 / \mathrm{mmr} .2012 .838$

\begin{abstract}
Liver-specific microRNA-122 (miR-122) is involved in the replication of hepatitis $\mathrm{C}$ virus $(\mathrm{HCV})$ and its potential as a target for antiviral intervention was recently assessed. However, the use of circulating miR-122 in the evaluation of liver function has never been reported. In the present study, changes of serum miRNA levels were first evaluated in acute human hepatotoxicity due to paraquat exposure. Serum samples were collected and analyzed using real-time reverse transcription PCR. The results showed a positive correlation between serum miR-122 and alanine aminotransferase, a clinical biomarker for liver function. Furthermore, serum miR-122 was assessed in patients with hepatitis B and hepatocarcinoma, resulting in distinct miR-122 profiles in these two closely related diseases. In addition to miR-122, another small RNA, U6 small nuclear RNA, was downregulated in hepatocarcinoma patients, suggesting its prognostic significance in this disease. Taken together, these lines of evidence indicate that serum miR-122 may provide a biomarker for diverse liver
\end{abstract}

Correspondence to: Dr Quan Du, Institute of Molecular Medicine, Peking University, Beijing 100871, P.R. China

E-mail: quan.du@pku.edu.cn

Dr Zhiyuan $\mathrm{Hu}$, State Key Laboratory of Proteomics, Beijing Proteome Research Center, Beijing 102206, P.R. China

E-mail: hu.zhiyuan@gmail.com

Dr Zicai Liang, Institute of Molecular Medicine, Peking University, Beijing 100871, P.R. China

E-mail: liangz@pku.edu.cn

Dr Xianfeng Ding, School of Life Science, Zhejiang Sci-Tech University, Hangzhou 310018, P.R. China

E-mail: bdd114@163.com

${ }^{*}$ Contributed equally

Key words: circulating microRNA, biomarker, liver injury diseases and, more importantly, suggest that a combination of nucleic acid biomarkers may be used as a sensitive and specific index for discriminating closely related diseases.

\section{Introduction}

Liver damage, as in the forms of hepatocyte carcinoma, viral hepatitis, acute hepatotoxicity or chronic hepatic injury, is frequently encountered in the practice of medicine. Accumulating knowledge in this field led to the development of a panel of protein-based blood biomarkers, including alanine aminotransferase (ALT), aspartate aminotransferase (AST), alkaline phosphatase (ALP), total bilirubin, direct bilirubin, total protein and albumin. Of these biomarkers, the blood ALT levels are the benchmark for liver function in clinical practice. Although liver malfunction is a common cause of elevated blood ALT levels, other factors impacting on this biomarker cannot be ignored. These effects occur mainly due to its extensive distribution in extrahepatic tissues, such as kidney, heart and skeletal muscle, which compromises its specificity in clinical practice. For example, patients with burns or muscle inflammation also show elevated blood ALT levels $(1,2)$, and approximately one-third of hepatitis $\mathrm{C}$ virus (HCV)-infected patients have a normal ALT reading in the blood (3).

Distinct from the distribution profile of ALT, a few liverspecific microRNAs (miRNAs), such as miR-122, have recently been characterized (4-6). miRNAs are a type of small endogenous non-coding RNA. Although the biological functions of miRNAs have yet to be elucidated, they are frequently dysregulated in human tumors, and tissue levels of specific miRNAs have been shown to correlate well with the pathological development of a variety of cancer types $(7,8)$. Furthermore, the latest finding of miRNA in the blood suggests the potential of miRNA-based blood biomarkers in cancer diagnosis $(9,10)$.

A previous study (11), using an acetaminophen-overdose liver injury model, determined miRNA expression profiles in mouse tissues and plasma. In comparison to a sham group, 
these authors found profound changes in the spectrum and expression levels of serum miRNAs in overdosed animals; certain miRNAs exhibited evident dose- and durationdependent alterations in the plasma, paralleling the changes of serum ALT levels and histological degeneration.

Similar to the acetaminophen-overdose mouse model, acute pesticide poisoning in human occurs after a single exposure, and results in severe injury to the liver, kidney and nervous system. The majority of the pesticide poisoning cases in China are caused by N,N'-dimethyl-4,4'-bipyridinium dichloride, under the trade name of paraquat. Paraquat is one of the most widely used herbicides in the world. When ingested, it becomes highly toxic to mammals, including humans.

Thus, the aim of the present study was to characterize the behavior of serum miRNAs in paraquat-exposed individuals and evaluate their changes in acute human hepatotoxicity due to paraquat exposure.

\section{Patients and methods}

Human sample collection and ethics statement. The study was conducted according to the principles approved by the Ethics Review Boards of Zhejiang Cancer Hospital (Hangzhou, China), and the 307 Hospitals (Beijing, China). Written informed consent was provided for sample collection and subsequent analysis. In total, 101 serum samples were collected from 19 healthy individuals (controls), 36 paraquat-exposed individuals, 28 patients with hepatocarcinoma and 18 patients with hepatitis B and analyzed in the study. The serum was stored at $-80^{\circ} \mathrm{C}$ after samples collection.

Measurement of serum miRNA levels using quantitative $R T-P C R$. PCR primers and probes were from RiboBio (Guangzhou, China).

Quantitative real-time reverse transcription PCR was performed using serum. Briefly, 20- $\mu$ 1 reverse transcriptase reactions containing $11 \mu \mathrm{l}$ serum, $2 \mu \mathrm{l}$ RT primer, $2 \mu \mathrm{l} \mathrm{dNTP}$ (10 $\mu \mathrm{M}$ each), $4 \mu \mathrm{l}$ 5X First-strand buffer, $0.4 \mu \mathrm{l}$ RNase inhibitor $(40 \mathrm{U} / \mu \mathrm{l})$ and $0.8 \mu \mathrm{l}$ TIANScript M-MLV (2,000 U/l). For the reverse transcription, the mixture was incubated at $25^{\circ} \mathrm{C}$ for $5 \mathrm{~min}, 42^{\circ} \mathrm{C}$ for $50 \mathrm{~min}$ and, finally, at $95^{\circ} \mathrm{C}$ for $5 \mathrm{~min}$. Real-time PCR was then performed using an Eppendorf Mastercycler ep realplex system. The 25- $\mu$ l PCR reaction included $2 \mu \mathrm{l}$ RT product, $2.5 \mu \mathrm{l}$ 10X HotMaster Taq Buffer, $0.5 \mu \mathrm{l}$ dNTP (2.5 mM each), $1 \mu \mathrm{l} \mathrm{SYBR-Green} \mathrm{(Invitrogen),}$ $0.5 \mu 1 \mathrm{miRNA}$ forward primer $(10 \mu \mathrm{M}), 0.5 \mu \mathrm{l}$ miRNA reverse primer $(10 \mu \mathrm{M})$ and $0.15 \mu \mathrm{l}$ HotMaster Taq DNA polymerase (2.5 U/ $\mu \mathrm{l})$ (Tiangen, China). The reactions were carried out at $95^{\circ} \mathrm{C}$ for $10 \mathrm{~min}$, followed by 40 cycles of $95^{\circ} \mathrm{C}$ for $15 \mathrm{sec}$ and $60^{\circ} \mathrm{C}$ for $1 \mathrm{~min}$.

Statistical analysis. The data were analyzed using Pearson's correlation coefficient and were presented as the means \pm SD. $\mathrm{P}<0.01$ was considered statistically significant.

\section{Results}

microRNAs assessed in the study. To characterize the behavior of serum miRNAs in paraquat-exposed individuals, four of the most regulated miRNAs identified in a mouse model were evaluated in human samples: miR-122, miR-192, miR-483 and miR-711. In the previous study of acetaminophen-overdosed mice (11), the serum levels of miR-122 and miR-192 were markedly elevated by $474-$ and 80 -fold, respectively, whereas the serum levels of miR-483 and miR-711 were decreased by 4 - and 13-fold, respectively. Using quantitative real-time reverse transcription PCR (qRT-PCR), the serum levels of these miRNAs were first measured in 8 healthy individuals. Comparable serum levels of the four human miRNAs were identified in healthy individuals (Fig. 1).

Serum microRNA behavior in paraquat-exposed individuals. The serum levels of the four miRNAs were measured in 8 paraquat-exposed individuals (Fig. 2A). Blood AST was included in the assay to indicate the degree of liver injury. In agreement with the acetaminophen-overdose study (11), serum miR-122 was markedly elevated in the paraquat-exposed human samples. Of the 8 samples with ALT activity ranging from 447 to $1,960 \mathrm{U} / 1$, parallel increases of serum miR-122 and ALT were found. In addition, the consistent downregulation of miR-483 and miR-711 occurred in human samples, as with the mouse study. This downregulation likely reflected the conservation of mammalian hepatotoxicity. However, miR-192, another upregulated miRNA in the mouse study, was downregulated by 1.6- to 7.6-fold in the human hepatotoxicity samples. Although the reason for this discrepancy is unclear, it may indicate an inter-species difference, or specific cell responses induced by the two different chemicals. Notably, the most profound changes in the miRNAs occurred in the sample with the highest ALT reading, indicating that they were associated with the degree of liver injury, at least to some extent.

Among the four miRNAs, miR-122 is the most promising biomarker candidate in that it is liver-specific. The disclosed association between serum miR-122 and ALT suggests its involvement in drug-induced acute hepatotoxicity in humans.

Elevation of serum miR-122 in paraquat-exposed individuals. To better understand the behavior of circulating miR-122 in acute hepatotoxicity, daily sampling was performed on a paraquat-exposed patient during therapy. As expected, a correlation was found in the downregulation of serum miR-122 and ALT (Fig. 2B). To corroborate this finding, a large-scale analysis was carried out with 36 paraquat-exposed samples. In addition to the consistent elevation of serum miR-122, a positive correlation between serum miR-122 and ALT was found in samples with ALT readings $>600 \mathrm{U} / \mathrm{l}$. Taken together, paraquat exposure led to a marked elevation of serum miR-122 levels, similar to the finding in the acetaminophen-overdosed mice. This evidence demonstrated that serum miR-122 levels correlate well with the status of liver function in acute drug-induced hepatotoxicity in humans. Although further investigation is required to understand the underlying mechanism, a correlation of serum miR-122 with high ALT levels showed that the miR-122 levels may be a real sign of substantial liver injury.

miR-122 profile in hepatitis $B$ and hepatocarcinoma patients. miRNA-122 is the most abundant miRNA species in the liver, accounting for $\sim 70 \%$ of the total miRNA population, and is essential for cholesterol and lipid metabolism (12-15). Previous studies found that miR-122 is also crucial for the accumulation 


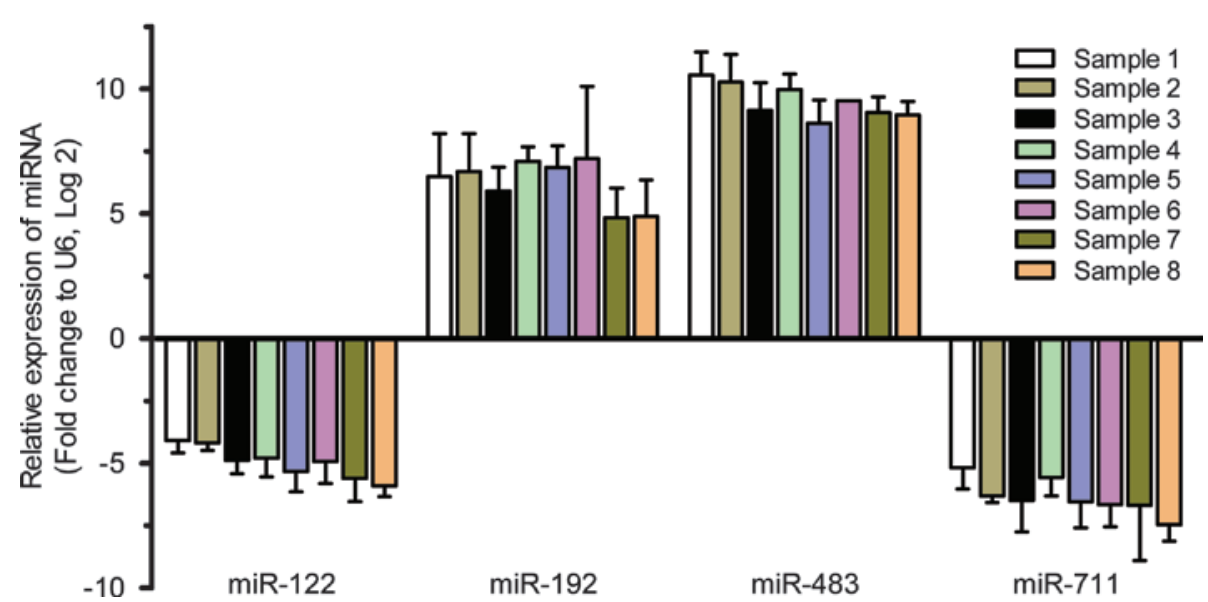

Figure 1. Serum levels of four human microRNAs measured using quantitative real-time RT-PCR in 8 serum samples collected from healthy individuals.

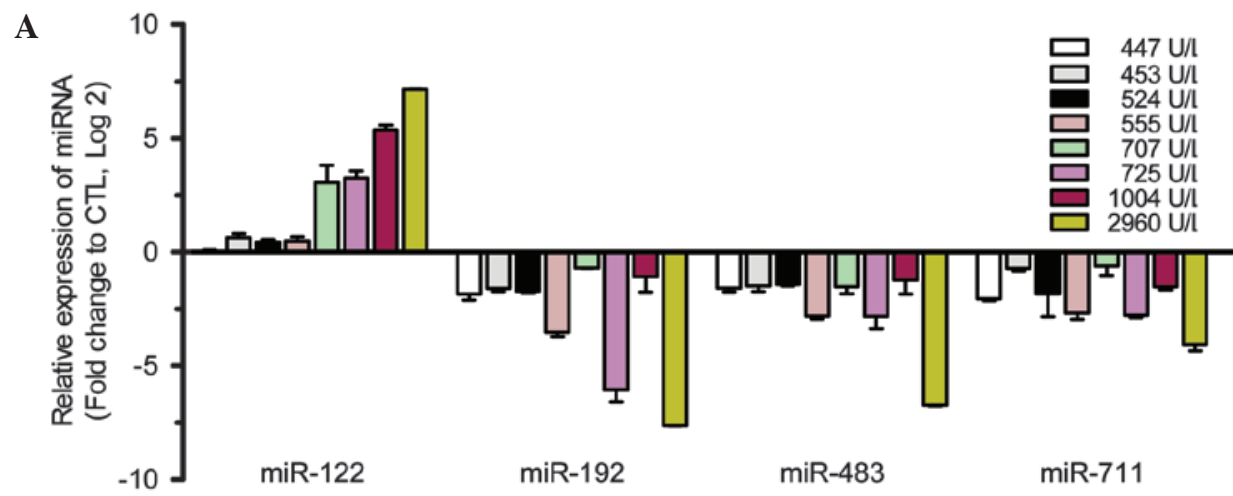

B

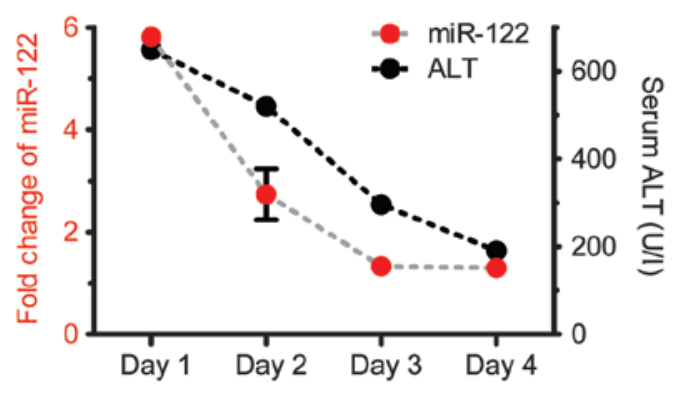

C

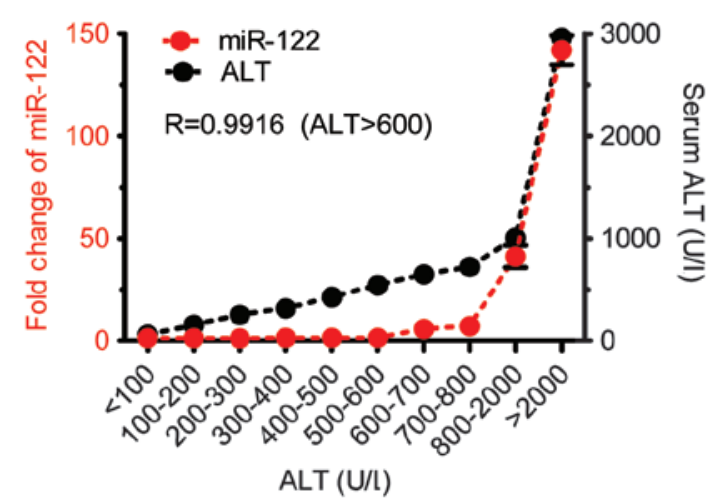

Figure 2. Correlation of serum miR-122 and ALT levels in paraquat-exposed individuals is shown. (A) Serum miRNA levels were measured using quantitative RT-PCR in 8 individuals exposed to a paraquat overdose. Calculated Pearson's correlation coefficients: miR-122, $R=0.9944$ and $P<0.0001 ; m i R-192, R=0.4658$ and $\mathrm{P}=0.2446$; miR-384, $\mathrm{R}=0.6442$ and $\mathrm{P}=0.0847$; miR-711, $\mathrm{R}=0.4747$ and $\mathrm{P}=0.2346$. (B) Comparison of serum miR-122 and ALT levels during therapy. The levels of miR-122 (red) are indicated on the left vertical axis, and the levels of ALT (black) are indicated on the right vertical axis. (C) Thirty-six paraquatexposed samples were divided into different ALT groups and the serum levels of miR-122 were quantified. The miR-122 levels (red) are indicated on the left vertical axis, and the ALT levels (black) are indicated on the right vertical axis. The experiments were repeated at least three times with two replicates in each assay. Data are expressed as the means \pm SD.

of HCV RNA in cultured liver cells, raising much interest in its role in viral intervention therapy $(4-6,13,16)$. To characterize the behavior of circulating miR-122 in patients with liver diseases, serum miR-122 levels were quantified in 18 individuals with hepatitis B and 28 with hepatocarcinoma (Fig. 3A and B). The range of ALT levels was from 29 to 1,321 U/1 in the hepatitis cases, and from 42 to 74 U/l. Serum miR-122 levels were determined by quantitative real-time RT-PCR. In contrast to the marked increase of serum miR-122 in hepatotoxicity cases, a milder elevation of miR-122 was found in hepatitis B cases, although the serum ALT for these cases was at a similar level. Approximately $40 \%$ of hepatitis patients showed a substantial downregulation of serum miR-122, whereas no apparent downregulation of miR-122 was found 
A

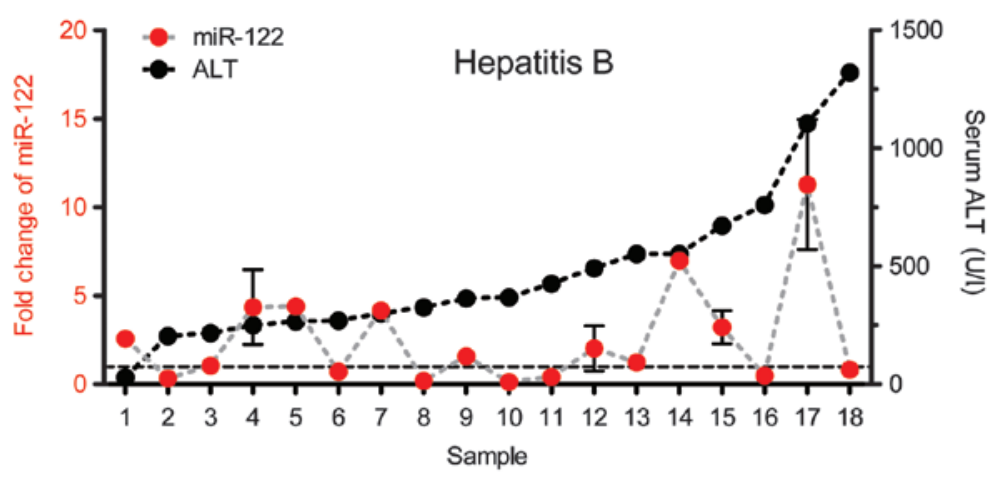

B

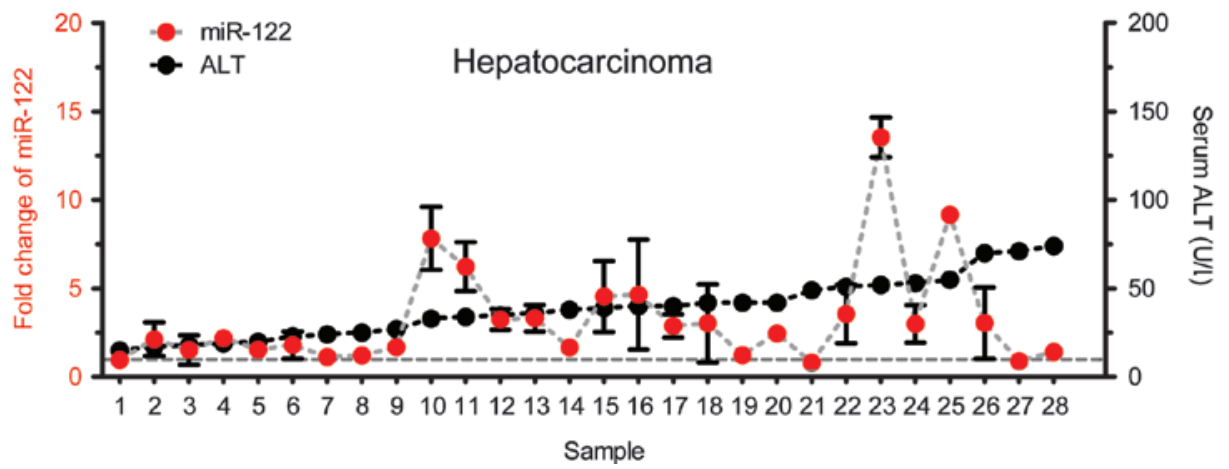

C

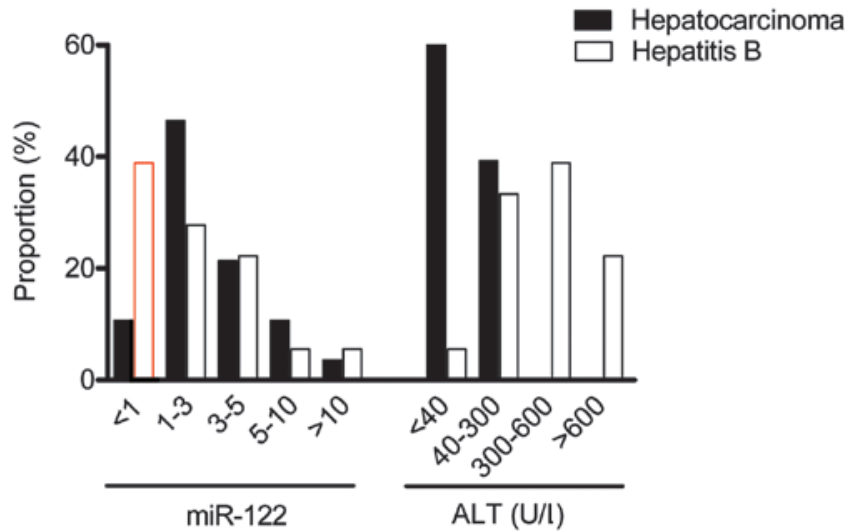

D

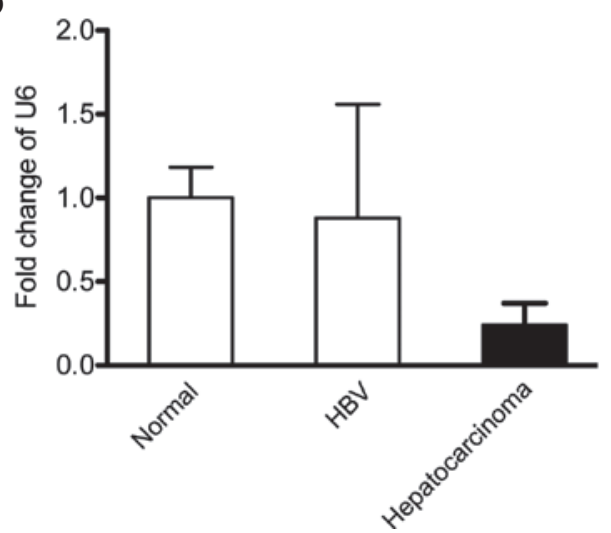

Figure 3. Serum miR-122 in individuals with hepatitis B or hepatocarcinoma. miR-122 levels were quantified in (A) 18 hepatitis B samples and (B) 28 hepatocarcinoma samples. The miR-122 levels (red) are indicated on the left vertical axis, and the ALT levels (black) are indicated on the right vertical axis. Experiments were repeated at least three times with two replicates in each assay; data are shown as the means \pm SD. (C) Distribution of hepatitis B and hepatocarcinoma cases in miR-122 and ALT groups. (D) Downregulation of U6 small small nuclear (sn)RNA in hepatocarcinoma cases. Serum levels of U6 snRNA were measured using quantitative RT-PCR and the levels were normalized to the healthy controls.

in hepatocarcinoma patients. Although the serum ALT in most hepatocarcinoma patients was within the normal range, a significant elevation of serum miR-122 was shown in $78 \%$ of patients, using a 1.5-fold increase as the cut-off. By contrast, only $50 \%$ of hepatitis patients showed upregulation (Fig. 3C).

Downregulation of serum U6 snRNA in hepatocarcinoma patients. In addition to miR-122, a marked decrease of serum U6 small nuclear RNA (snRNA) levels was also found in hepatocarcinoma patients. Compared to the healthy controls, an average 5-fold decrease of serum U6 snRNA was found in the hepatocarcinoma, but not in the hepatitis patients (Fig. 3D). U6 is a non-coding RNA component of U6 snRNP, a major effector molecule involved in RNA splicing. The sequence of U6 RNA is highly conserved across species from yeast to mammals
(17), and is frequently used as a housekeeping control in gene expression assays. Although the regulated expression of U6 snRNA was recently reported in 13 normal human tissues (18), neither U6 snRNA nor circulating U6 snRNA have previously been associated with human carcinomas in published studies Therefore, the downregulation found in serum U6 snRNA in hepatocarcinoma patients provided the first evidence that this so-called housekeeping control may be involved in tumorigenesis and may be used as a specific tumor biomarker.

\section{Discussion}

Liver-specific mir-122 is known to be involved in replicating HCV. Moreover, characteristic small RNA profiles have been identified in diverse hepatic diseases. In the present study, 
changes of serum miRNA levels were evaluated in acute human hepatotoxicity due to paraquat exposure, to assess the use of circulating miR-122 in the liver function study.

Results of the present study showed that paraquat exposure led to a marked elevation of serum miR-122 levels, which was similar to the finding in the acetaminophen-overdosed mice. This finding shows that serum miR-122 levels correlate well with the status of liver function in acute drug-induced hepatotoxicity in humans. Additionally, while a marked elevation of the serum miR-122 levels was found in cases of acute drug-induced hepatotoxicity, the change of miR-122 was much milder in hepatitis and hepatocarcinoma patients. Despite differing extents, a general elevation of serum miR-122 confirmed the hepatocyte injury in these cases. In addition, a decrease of U6 snRNA in hepatocarcinoma patients showed that the serum levels of other small RNA species may also be regulated under specific physiological or pathological conditions. Although further studies are required to verify the clinical significance of miR-122, the distinct small RNA profiles revealed in diverse hepatic diseases make it possible to finely map the cause of liver injury, and lead us to speculate that a combination of small RNA biomarkers instead of a single biomarker may be more sensitive and specific than the current tests for discrimination of the status of liver function and injury.

Therefore, bearing in mind that a candidate approach rather than a systematic approach has been employed as well as the potential inter-species differences, the present study provides a starting point for the comprehensive profiling of miRNA changes in diverse hepatic diseases.

\section{Acknowledgements}

This study was supported by the Guangdong Science and Technology Department (2011B090400478), the National High-tech R\&D Program of China (2007AA02Z165), the National Natural Science Foundation of China (30873187, 30771085 and 30871385), the Zhejiang Natural Science Foundation(Y2100681),theBeijing Natural ScienceFoundation (5092011), the National Drug Program (2009ZX09503) SKLPK200903 and the National Basic Research Program of China (2007CB512100).

\section{References}

1. Halkes S, van den Berg A, Hoekstra M, du Pont J and Kreis R: Transaminase and alkaline phosphatase activity in the serum of burn patients treated with highly purified tannic acid. Burns 28: 449-453, 2002.

2. Korones DN, Brown MR and Palis J: 'Liver function tests' are not always tests of liver function. Am J Hematol 66: 46-48, 2001.

3. Dufour DR, Lott JA, Nolte FS, Gretch DR, Koff RS and Seeff LB: Diagnosis and monitoring of hepatic injury. II. Recommendations for use of laboratory tests in screening, diagnosis, and monitoring. Clin Chem 46: 2050-2068, 2000.

4. Jopling CL, Yi M, Lancaster AM, Lemon SM and Sarnow P: Modulation of hepatitis C virus RNA abundance by a liverspecific MicroRNA. Science 309: 1577-1581, 2005.

5. Randall G, Panis M, Cooper JD, et al: Cellular cofactors affecting hepatitis $\mathrm{C}$ virus infection and replication. Proc Natl Acad Sci USA 104: 12884-12889, 2007.

6. Lanford RE, Hildebrandt-Eriksen ES, Petri A, et al: Therapeutic silencing of microRNA-122 in primates with chronic hepatitis C virus infection. Science 327: 198-201, 2010.

7. Lee YS and Dutta A: MicroRNAs in cancer. Annu Rev Pathol 4: 199-227, 2009

8. Dillhoff M, Wojcik SE and Bloomston M: MicroRNAs in solid tumors. J Surg Res 154: 349-354, 2009.

9. Chen X, Ba Y, Ma L, et al: Characterization of microRNAs in serum: a novel class of biomarkers for diagnosis of cancer and other diseases. Cell Res 18: 997-1006, 2008.

10. Mitchell PS, Parkin RK, Kroh EM, et al: Circulating microRNAs as stable blood-based markers for cancer detection. Proc Natl Acad Sci USA 105: 10513-10518, 2008.

11. Wang K, Zhang S, Marzolf B, Troisch P, Brightman A, Hu Z, Hood LE, et al: Circulating microRNAs, potential biomarkers for drug-induced liver injury. Proc Natl Acad Sci USA 106: 4402-4407, 2009.

12. Esau C, Davis S, Murray SF, et al: miR-122 regulation of lipid metabolism revealed by in vivo antisense targeting. Cell Metab 3: 87-98, 2006.

13. Krützfeldt J, Rajewsky N, Braich R, Rajeev KG, Tuschl T, Manoharan M and Stoffel M: Silencing of microRNAs in vivo with 'antagomirs'. Nature 438: 685-689, 2005.

14. Elmén J, Lindow M, Schütz S, et al: LNA-mediated microRNA silencing in non-human primates. Nature 452: 896-899, 2008

15. Lagos-Quintana M, Rauhut R, Yalcin A, Meyer J, Lendeckel W and Tuschl T: Identification of tissue-specific microRNAs from mouse. Curr Biol 12: 735-739, 2002.

16. Sarasin-Filipowicz M, Krol J, Markiewicz I, Heim MH and Filipowicz W: Decreased levels of microRNA miR-122 in individuals with hepatitis $C$ responding poorly to interferon therapy. Nat Med 15: 31-33, 2009.

17. Brow DA and Guthrie C: Spliceosomal RNA U6 is remarkably conserved from yeast to mammals. Nature 334: 213-218, 1988

18. Peltier HJ and Latham GJ: Normalization of microRNA expression levels in quantitative RT-PCR assays: identification of suitable reference RNA targets in normal and cancerous human solid tissues. RNA 14: 844-852, 2008. 